\title{
The STAFF III Database: ECGs Recorded During Acutely Induced Myocardial Ischemia
}

\author{
Juan Pablo Martínez ${ }^{1,2}$, Olle Pahlm ${ }^{3}$, Michael Ringborn ${ }^{4}$, Stafford Warren ${ }^{5}$, Pablo Laguna ${ }^{1,2}$, \\ Leif Sörnmo 6 \\ ${ }^{1}$ BSICoS group, I3A Research Institute and IIS Aragón, University of Zaragoza, Zaragoza, Spain \\ ${ }^{2}$ Biomedical Research Networking Center (CIBER-BBN), Zaragoza, Spain \\ ${ }^{3}$ Department of Clinical Sciences, Lund University, and Lund University Hospital, Sweden \\ ${ }^{4}$ Thoracic Center, Blekingesjukhuset, Karlskrona, Sweden \\ ${ }^{5}$ Anne Arundel Medical Center, Annapolis, USA \\ ${ }^{6}$ Department of Biomedical Engineering and CIEL Center, Lund University, Lund, Sweden
}

\begin{abstract}
The STAFF III database was acquired with the aim of better understanding the ECG signatures observed during acute ischemia, with special focus on high-frequency $Q R S$ components. The database contains recordings from 104 patients undergoing elective balloon percutaneous coronary intervention. The database has not only been analyzed in numerous clinical studies, but also turned out to be an excellent tool for methodological development. Its use has, by far, exceeded the original aim. Inspired by this fact, the database has now been made publicly available at Physionet.
\end{abstract}

\section{Introduction}

The STAFF III database was acquired in 1995-96 at the Charleston Area Medical Center (WV, USA), where single prolonged balloon inflation had been introduced to achieve optimal results of the elective balloon percutaneous coronary intervention (PCI) procedure, replacing the typical series of brief inflations. The database consists of ECG recordings from 104 patients, thus accounting for substantial inter-patient variability due to prolonged balloon inflation (before the stent era) as well as variability in rhythm and waveform morphology; Fig. 11 illustrates changes observed during balloon inflation. Only patients receiving elective balloon PCI in one of the major coronary arteries were included. Patients suffering from ventricular tachycardia, undergoing an emergency procedure, or demonstrating signal loss during acquisition, were excluded. The database was originally acquired for analyzing high-frequency QRS components during acute ischemia [1]. A description of the context and history of the database is found in [3], and a review of methodological development in [4]. The database is publicly available at
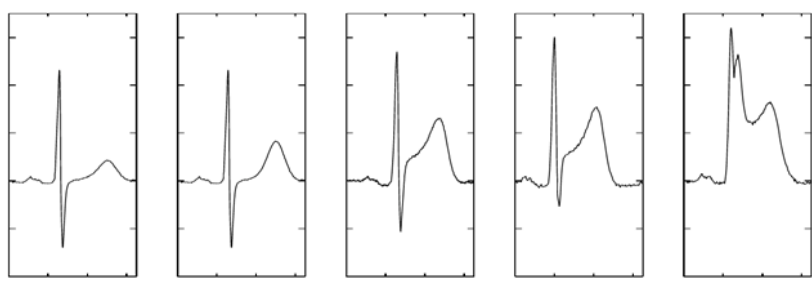

Figure 1. From left to right: Sequential ECG changes observed during the course of myocardial ischemia.

Physionet (identified by the doi:10.13026/C20P4H) [2].

\section{Data Acquisition}

The occlusion procedure was defined as follows. Preinflation (baseline) ECGs were acquired for $5 \mathrm{~min}$ at rest in supine position in either the patientt's room at the ward, in the catheterization laboratory, or both, prior to any catheter insertion. Inflation ECGs were acquired during a single, or in some cases multiple (up to five times), balloon inflations in each patient. The mean inflation time was $4 \min 23 \mathrm{~s}$, ranging from $1 \mathrm{~min} 30 \mathrm{~s}$ to $9 \mathrm{~min}$ and $54 \mathrm{~s}$. In 86 inflations, the recording was started some time before balloon inflation (4 to 205 s). Moreover, in some cases, the recording was continued after balloon deflation, with a postinflation period $>60 \mathrm{~s}$ in all but 11 inflations. All time instants related to balloon inflation/deflation were manually annotated. Post-inflation ECGs were acquired for $5 \mathrm{~min}$ at rest in supine position in either the cath lab or the patientt's room at the ward, or both.

The database contains a total of 152 occlusions in the major coronary arteries, distributed as 58 occlusions in the left anterior descending (LAD) artery, 59 in the right coronary artery (RCA), 32 in the left circumflex artery (LCX), 


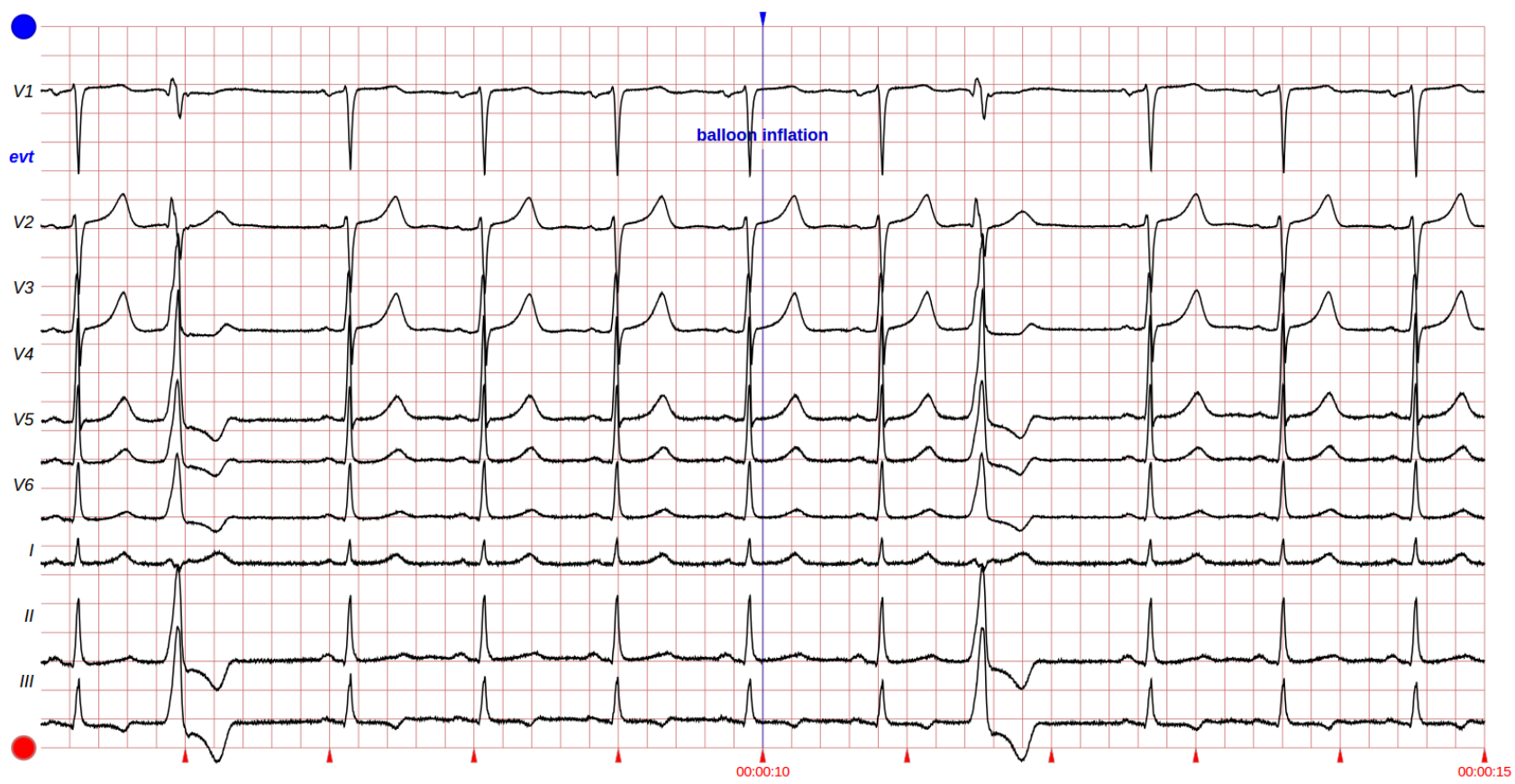

Figure 2. ECG with the annotated onset of balloon inflation.

and 3 in the left main (LM) artery. Based on ECG criteria, 35 patients had previous myocardial infarction.

The database consists of standard 12-lead ECG data. Standard electrode placements were used for the precordial leads, whereas the limb leads were obtained with the Mason-Likar electrode configuration to reduce noise originating from skeletal muscle. Data acquisition was based on custom-made equipment by Siemens-Elema AB, offering an extraordinary input amplitude range. The ECG was digitized at a sampling rate of $1000 \mathrm{~Hz}$ and an amplitude resolution of $0.625 \mu \mathrm{V}$. These specifications ensured that high-resolution digital signals could be produced so that high-frequency components, as well as other subtle electrophysiological phenomena, could be analyzed.

Dye injections during catheterization and angiography may cause changes in ECG morphology, and therefore injections were annotated. Since far from all injections were annotated, database users are advised to be cautious when brief changes in the ECG are observed that mimic the dynamics of the known dye injections.

\section{Data Files}

The clinical information and annotations are included in a companion spreadsheet, see Table $\mathbb{T}$ for details on data types. The time instants of balloon inflation/deflation and the contrast injection are also provided as annotator files.

Each recording is defined by the data files *.dat and *.hea, together with the annotation file *.event which includes the time instants of balloon inflation/deflation and contrast injection. Figure $\square$ displays an ECG with the annotated onset of balloon inflation.

In some files, additional inflations have been identified not originally indicated. These additional inflations are included in the *.event file.

For records containing more than one inflation, the annotated time until inflation (D0), for the second and subsequent inflations, is not strictly the time at which the balloon is inflated, as D0 includes the duration of the previous preinflation(s), inflation(s), and post-inflation(s), see example in Table $\mathbb{\text { I. }}$.

\section{Clinical Applications}

Coronary artery occlusion reduces myocardial blood flow and thus induces ischemia locally in the affected myocardial area. This ischemia most often produces considerable change the ECG morphology. The changes are explored for detecting, monitoring, and quantifying ischemia. While the ST level is the most widely used index, it is known to suffer from poor performance when used for detecting ischemia, thereby limiting its usefulness in clinical practice.

The number of studies involving the STAFF III database has increased considerably over the years, as it has been found highly adequate for several other research problems than high-frequency QRS analysis. Although the original motivation for acquiring the database was clinically oriented, the database has been found most valuable for developing, improving, and evaluating a wide range of signal 
Table 1. Data types

\begin{tabular}{|c|c|}
\hline Data & Example record \#39 \\
\hline Record \# & 39 \\
\hline Age (years) & 48 \\
\hline $\operatorname{Sex}(\mathrm{m} / \mathrm{f})$ & $m$ \\
\hline Baseline record at room (BR) & $39 a$ \\
\hline $\begin{array}{l}\text { First baseline record } \\
\text { at cath-lab }(\mathrm{BC} 1)\end{array}$ & $39 b$ \\
\hline $\begin{array}{l}\text { Second baseline record } \\
\text { at cath-lab }(\mathrm{BC} 2)\end{array}$ & - \\
\hline First inflation record (BI1) & $39 c$ \\
\hline Occluded artery in record BI1 & mid LAD \\
\hline Occlusion time (in s.) & 0 \\
\hline Inflation duration (in s.) & 285 \\
\hline Reperfusion duration at BI1 (in s.) & 7 \\
\hline Contrast injection at BI1 & - \\
\hline Second inflation record (BI2) & $39 c$ \\
\hline Occluded artery in record $\mathrm{BI} 2$ & prox LAD \\
\hline Occlusion time (in s.) & 292 \\
\hline Inflation duration(in s.) & 200 \\
\hline Reperfusion duration at $\mathrm{BI} 2$ (in s.) & 30 \\
\hline Contrast injection at $\mathrm{BI} 2$ & - \\
\hline Third inflation record (BI3) & $39 c$ \\
\hline Occluded artery in record BI3 & prox LAD \\
\hline Occlusion time (in s.) & 522 \\
\hline Inflation duration (in s.) & 137 \\
\hline Reperfusion duration at BI3 (in s.) & 106 \\
\hline Contrast injection at $\mathrm{BI} 3$ & $502 ; 707$ \\
\hline Fourth inflation record (BI4) & $39 d$ \\
\hline Occluded artery in record BI4 & prox LAD \\
\hline Occlusion time (in s.) & 0 \\
\hline Inflation duration (in s.) & 152 \\
\hline Reperfusion duration at BI4 (in s.) & 60 \\
\hline Fifth inflation record (BI5) & $39 d$ \\
\hline Occluded artery in record BI5 & prox LAD \\
\hline Occlusion time (in s.) & 212 \\
\hline Inflation duration (in s.) & 117 \\
\hline reperfusion duration at BI5 (in s.) & 36 \\
\hline First post-inflation record at cath-lab (PC1) & - \\
\hline Second post-inflation record at cath-lab (PC2) & - \\
\hline First post-inflation record at room (PR1) & $39 e$ \\
\hline Second post-inflation record at room (PR2) & - \\
\hline Prior MI location & anterior \\
\hline
\end{tabular}

processing techniques [4].

Considerable research has been done on the database, including the following clinical aspects:

- Time course of ischemia-induced changes in repolarization indices. Figure B illustrates the ST level before, during, and after balloon inflation.

- Time course of ischemia-induced changes in indices describing ventricular depolarization, and evaluation of variability/reproducibility across patients. Figure 4 illustrates QRS upslope during control (two recordings), occlusion, and reperfusion. The reperfusion effects after artery reopening and/or contrast injections can be observed.

- Spatial distribution of ischemic markers by analysing their signature at different leads. Eventually this can be correlated to the occluded artery. Figure 5 illustrates the variability in QRS up- and downslope for different leads.

- Implications of myocardial ischemia on $\mathrm{T}$ wave alter-

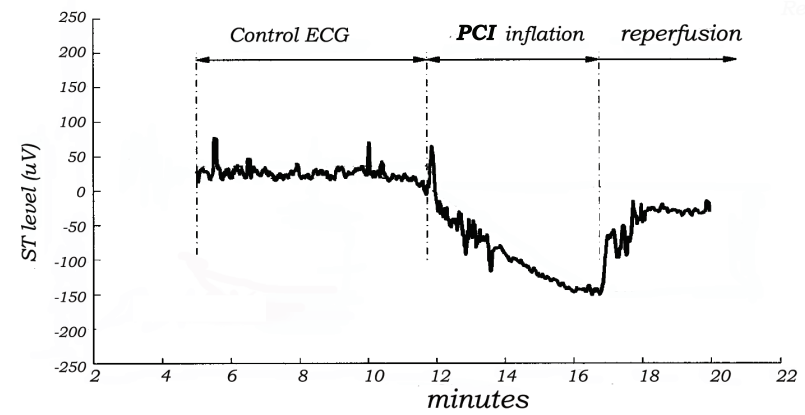

Figure 3. ST level dynamics during control, occlusion, and reperfusion (adapted from [5]).
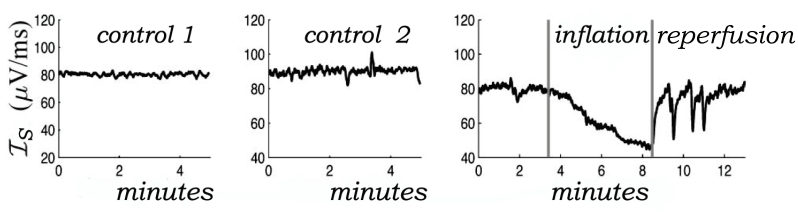

Figure 4. QRS upslope $\left(\mathcal{I}_{\mathcal{S}}\right)$ dynamics during control, and occlusion/reperfusion (adapted from [6]).

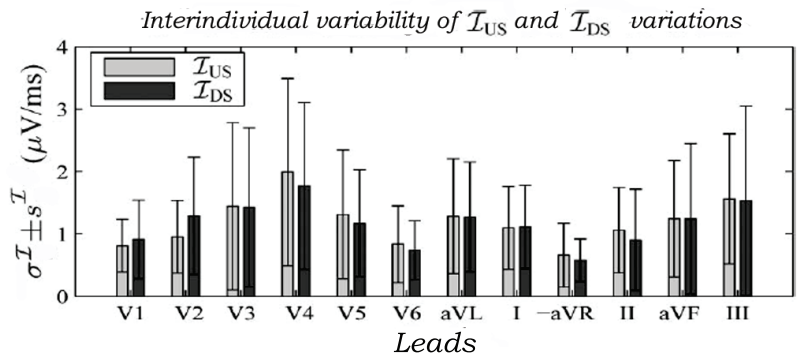

Figure 5. Variability in QRS up- and downslope across leads (adapted from [6]).

nans. Figure $\mathbf{6}$ illustrates the dynamics of repolarization alternans amplitude and ST level.

- Ischemia detection exploring the stronger non-linear response of QRS angles to ischemia. Figure 7 illustrates QRS angle and ST dynamics.

\section{Contributors}

The primary investigator Dr. Stafford Warren designed the study protocol together with Dr. Galen Wagner at Duke University Medical Center (Durham, NC, USA); Dr. Michael Ringborn was responsible for data acquisition. The database has been distributed by Prof. Leif Sörnmo, who was responsible for the acquisition equipment and software.

Dr. Wagner was the leader of the STAFF studies, and promoter of the clinical design and the analysis performed 

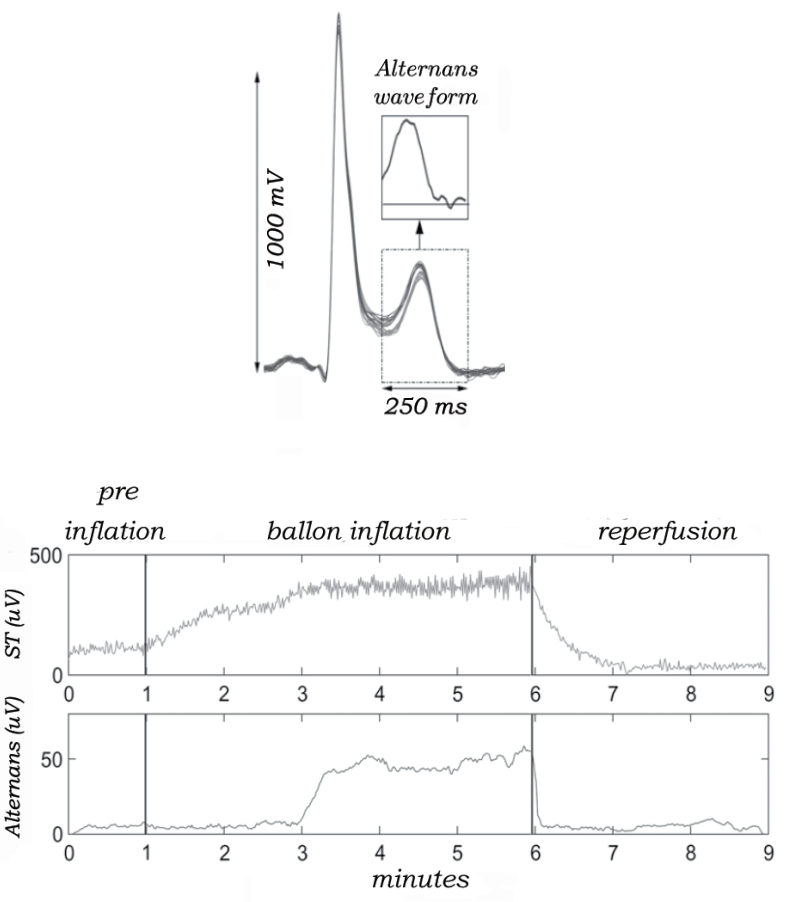

Figure 6. Repolarization alternans and ST level before, during, and after balloon inflation (adapted from [4] ).

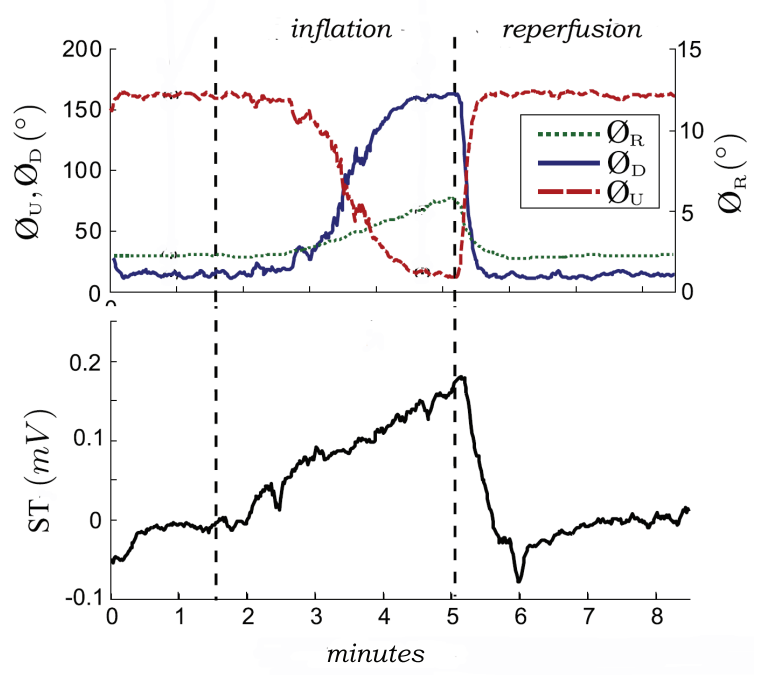

Figure 7. QRS angles (upwards $\emptyset_{U}$, downwards $\emptyset_{D}$, and middle $\emptyset_{R}$ ) and ST level (adapted from [7]]).

on the data. He was a strong proponent of making the database public.

\section{Conclusions}

The STAFF III Database is now publicly available on Physionet to stimulate research on transient phenomena in the ECG associated with acute myocardial ischemia. The database is annotated with respect to the important characteristics of acute ischemia, i.e., the onset/end of balloon inflation and the occluded artery. The ischemia so generated corresponds to total occlusion. The time for contrast injection is also included, allowing the evaluation of contrastinduced effects on the ECG. Control recordings before and after occlusion are included. The database offers an excellent testbed for evaluating methods and techniques aimed at quantifying myocardial ischemia.

\section{Acknowledgements}

This work was supported in part by project DPI201675458-R from Spanish Ministry of Economy and Competitiveness (MINECO), Spain, and by Aragón Government, Spain, and from European Social Fund (EU) to the BSICoS group.

\section{References}

[1] J. Pettersson, E. Carro, L. Edenbrandt, O. Pahlm, M. Ringborn, L. Sörnmo, S. Warren, G. Wagner. Spatial, individual and temporal variation of the high frequency QRS amplitudes in the 12 standard electrocardiographic leads. Am Heart J, vol. 139, pp.352-358, 2000.

[2] A. Goldberger, L. Amaral, L. Glass, J. Hausdorff, P. Ivanov, R. Mark, J. Mietus, G. Moody, C. Peng, H. Stanley. PhysioBank, PhysioToolkit, and PhysioNet: Components of a New Research Resource for Complex Physiologic Signals. Circulation vol. 101, pp. e215-e220, 2000;

[3] S. Warren, G. Wagner. The STAFF studies of the first 5 minutes of percutaneous coronary angioplasty balloon occlusion in man. J Electrocardiol vol. 47, pp. 402-407, 2014.

[4] P. Laguna, L. Sörnmo. The STAFF III ECG database and its significance in methodological development and evaluation. J Electrocardiol, vol. 47, pp. 408-417, 2014.

[5] J. García, P. Lander, L. Sörnmo, S. Olmos, G. Wagner, P. Laguna. Comparative study of local and Karhunen-Loèvebased ST-T indexes in recordings from human subjects with induced myocardial ischemia. Comput Biomed Res, vol. 31, pp.271-292, 1998.

[6] D. Romero, M. Ringborn, P. Laguna, O. Pahlm, E. Pueyo. Depolarization changes during acute myocardial ischemia by evaluation of QRS slopes. Standard lead and vectorial approach. IEEE Trans Biomed Eng vol. 58, pp. 110-120, 2011.

[7] D. Romero, M. Ringborn, P. Laguna E. Pueyo. Detection and quantification of acute myocardial ischemia by morphologic evaluation of QRS changes by an angle-based method. J Electrocardiol vol. 46, pp. 204-214, 2013.

Address for correspondence:

Juan Pablo Martínez, Campus Río Ebro, Ada Byron Building, c $\backslash$ Maria de Luna, 1, 50018 Zaragoza (Spain),

jpmart@unizar.es 